\title{
A REMARK ON TRANS-SASAKIAN 3-MANIFOLDS
}

\author{
YANING WANG AND WENJIE WANG
}

\begin{abstract}
Let $M$ be a trans-Sasakian 3-manifold of type $(\alpha, \beta)$. In this paper, we give a negative answer to the question proposed by S. Deshmukh [Mediterr. J. Math. 13 (2016), no. 5, 2951-2958], namely we prove that the differential equation $\nabla \beta=\xi(\beta) \xi$ on $M$ does not necessarily imply that $M$ is homothetic to either a Sasakian or cosymplectic manifold even when $M$ is compact. Many examples are constructed to illustrate this result.
\end{abstract}

\section{INTRODUCTION}

A trans-Sasakian manifold $M$ is an almost contact metric manifold such that the product $M \times \mathbb{R}$ belongs to the class $W_{4}$ of Hermitian manifolds (see [23]). Hermitian manifolds of class $W_{4}$ are closely related to locally conformally Kähler manifolds (see [13]). In [18, Marrero gave the local structures of trans-Sasakian manifolds, namely a connected trans-Sasakian manifold of dimension greater than three must be of class $C_{5}$ or $C_{6}$. A trans-Sasakian manifold is said to be proper if it is one of the above two cases. However, there exist many trans-Sasakian 3-manifolds which are not proper (see [3, 20]), namely neither $\alpha$ nor $\beta$ is zero. Therefore, to find on what condition a trans-Sasakian 3-manifold is proper is an interesting problem. Recently, S. Desmukh et al. in [8, 9, 10, 11, 12, obtained various conditions under which a compact trans-Sasakian 3-manifold is homothetic to either a Sasakian or a cosymplectic 3-manifold. Trans-Sasakian 3-manifolds under some curvature restrictions were also studied by U. C. De et al. in [5, 6, 7] and Wang [24].

In [8, without the compactness assumption, Deshmukh proved

Theorem 1.1 ([8]). If a connected trans-Sasakian 3-manifold $M$ of type $(\alpha, \beta)$ satisfies $\nabla \alpha=\xi(\alpha) \xi$ with $\alpha \neq 0$, then $M$ is homothetic to a Sasakian manifold.

In the proof of the above theorem, Deshmukh found that $\nabla \alpha=\xi(\alpha) \xi$ and $\alpha \neq 0$ imply $\nabla \beta=\xi(\beta) \xi$. In view of similar but different properties of $\alpha$ and $\beta$, the author in [8] proposed the following interesting question:

2010 Mathematics Subject Classification. Primary 53D15; Secondary 53C25.

Key words and phrases. Compact trans-Sasakian 3-manifold; $\mathcal{D}$-homothetic deformation; $\alpha$ Sasakian manifold; $\beta$-Kenmotsu manifold.

The authors were supported by the Youth Science Foundation of Henan Normal University (No. 2014QK01). 
Question 1.1. Does the differential equation $\nabla \beta=\xi(\beta) \xi$ give a result similar to Theorem 1.1 or not?

The above question is equivalent to asking if differential equation $\nabla \beta=\xi(\beta) \xi$ on a trans-Sasakian 3-manifold implies that the manifold is homothetic to either a Sasakian or cosymplectic 3-manifold. In this paper, we aim to investigate this question and give a negative answer. Namely, we prove

Theorem 1.2. If a connected compact trans-Sasakian 3-manifold of type $(\alpha, \beta)$ satisfies $\nabla \beta=\xi(\beta) \xi$, then we have either $\alpha=0$ or $\beta=0$. However, the vanishing of $\alpha$ or $\beta$ does not necessarily imply that the other one is a constant.

After giving proof of the above Theorem 1.2 in Section 3, we construct many concrete examples to illustrate our main results.

\section{TRANS-SASAKIAN MANIFOLDS}

According to D. E. Blair [2], an almost contact metric structure defined on a smooth differentiable manifold $M$ of dimension $2 n+1$ is a $(\phi, \xi, \eta, g)$-structure satisfying

$$
\begin{gathered}
\phi^{2}=-\mathrm{id}+\eta \otimes \xi, \quad \eta(\xi)=1, \\
\phi^{*} g=g-\eta \otimes \eta,
\end{gathered}
$$

where $\phi$ is a $(1,1)$-type tensor field, $\xi$ is a tangent vector field called the characteristic or the Reeb vector field, and $\eta$ is a 1-form called the almost contact form. A Riemannian manifold $M$ furnished with an almost contact metric structure is said to be an almost contact metric manifold, denoted by $(M, \phi, \xi, \eta, g)$.

Let $M$ be an almost contact metric manifold of dimension $2 n+1$. On the product $M \times \mathbb{R}$ there exists an almost complex structure $J$ defined by

$$
J\left(X, f \frac{\mathrm{d}}{\mathrm{d} t}\right)=\left(\phi X-f \xi, \eta(X) \frac{\mathrm{d}}{\mathrm{d} t}\right),
$$

where $X$ denotes a vector field tangent to $M^{2 n+1}, t$ is the coordinate of $\mathbb{R}$ and $f$ is a $\mathcal{C}^{\infty}$-function on $M^{2 n+1} \times \mathbb{R}$. An almost contact metric manifold is said to be normal if the above almost complex structure $J$ is integrable and this is equivalent to $[\phi, \phi]=-2 \mathrm{~d} \eta \otimes \xi$, where $[\phi, \phi]$ denotes the Nijenhuis tensor of $\phi$.

An almost contact metric manifold is said to be a trans-Sasakian manifold (see [18]) if it is normal and $\mathrm{d} \eta=\alpha \Phi, \mathrm{d} \Phi=2 \beta \eta \wedge \Phi$, where $\alpha=\frac{1}{2 n} \operatorname{tr}(\phi \nabla \xi), \beta=\frac{1}{2 n} \operatorname{div} \xi$ and $\Phi(\cdot, \cdot)=g(\cdot, \phi \cdot)$. It is known (see [3] ) that an almost contact metric manifold $M$ is trans-Sasakian if and only if there exist two smooth functions $\alpha$ and $\beta$ satisfying

$$
\left(\nabla_{X} \phi\right) Y=\alpha(g(X, Y) \xi-\eta(Y) X)+\beta(g(\phi X, Y) \xi-\eta(Y) \phi X)
$$

for any vector fields $X$ and $Y$.

A trans-Sasakian manifold is denoted by $(M, \phi, \xi, \eta, \alpha, \beta)$ and is called a transSasakian manifold of type $(\alpha, \beta)$. From the definition of tran-Sasakian manifolds, putting $Y=\xi$ in $(2.2)$ and using (2.1) we have

$$
\nabla_{X} \xi=-\alpha \phi X+\beta(X-\eta(X) \xi)
$$


for any vector field $X$.

By Propositions 1 and 2 and Corollary 1 of [19], we observe that a normal almost contact metric 3-manifold is always trans-Sasakian. Therefore, by the definition of trans-Sasakian manifolds, we state that an almost contact metric 3-manifold is trans-Sasakian if and only if it is normal.

\section{SOME REMARKS ON TRANS-SASAKIAN 3-MANIFOLDS}

Before giving the proof of our main results, firstly we must clarify some important concepts that are easily confused.

As seen in the Introduction, a trans-Sasakian 3-manifold of type $(\alpha, \beta)$ is an $\alpha$-Sasakian manifold (see [14) if $\alpha \in \mathbb{R}^{*}$ and $\beta=0$; or a $\beta$-Kenmotsu manifold if $\beta \in \mathbb{R}^{*}$ and $\alpha=0$ (see [14]); or a cosymplectic manifold if $\alpha=\beta=0$ (see [2]). An $\alpha$-Sasakian manifold becomes a Sasakian manifold (see [2]) if $\alpha=1$. Similarly, a $\beta$-Kenmotsu manifold becomes a Kenmotsu manifold (see [15]) if $\beta=1$.

A trans-Sasakian manifold of type $(\alpha, \beta)$ is of $C_{6}$-class if $\beta=0$ (see [4]). As seen in [18, Lemma 3.1], $\alpha$, on a trans-Sasakian manifold of $C_{6}$-class of dimension greater than three, is a constant. Then the trans-Sasakian manifolds of $C_{6}$-class of dimension greater than 3 are just $\alpha$-Sasakian manifolds (see [14]). However, $\alpha$ on a trans-Sasakian 3-manifold of $C_{6}$-class is not necessarily a constant even when the manifold is compact. The proof for this is given after proving Theorem 3.1 .

A trans-Sasakian manifold of type $(\alpha, \beta)$ is of $C_{5}$-class if $\alpha=0$ (see [4]). On such manifolds of dimension greater than three there holds naturally $\mathrm{d} \beta \wedge \eta=0$ (see [22]), or equivalently, $\nabla \beta=\xi(\beta) \xi$. However, the above equation does not necessarily hold for dimension three. The set of all $\beta$-Kenmotsu manifolds is a proper subset of that of all trans-Sasakian manifolds of $C_{5}$-class. For trans-Sasakian manifolds of $C_{5}$-class with non-constant function $\beta$ we refer the reader to [1, 3, 15, 22. Note that a trans-Sasakian manifold of $C_{5}$-class is also called a $f$-cosymplectic manifold (see [1]) or an f-Kenmotsu manifold (see [16, 22, 25]). In this paper, in order to answer Question 1.1 we also construct some compact trans-Sasakian 3-manifolds of $C_{5}$-class with non-constant function $\beta$.

The following lemma was proved in [7] (see also [12]).

Lemma 3.1 ([7, Theorem 3.2]). On a trans-Sasakian 3-manifold of type $(\alpha, \beta)$ we have

$$
\xi(\alpha)+2 \alpha \beta=0 .
$$

In this paper, we denote by $\nabla f$ the gradient of a smooth function $f$ on $M$. Moreover, putting $n=1$ in [7, Proposition 3.4] we obtain the following lemma.

Lemma 3.2 ([7, Proposition 3.4]). On a trans-Sasakian 3-manifold of type $(\alpha, \beta)$ we have

$$
Q \xi=\phi(\nabla \alpha)-\nabla \beta+\left(2\left(\alpha^{2}-\beta^{2}\right)-\xi(\beta)\right) \xi
$$

where $Q$ denotes the Ricci operator associated with the Ricci tensor $S$ which is defined by $S(\cdot, \cdot)=\operatorname{trace}\{X \rightarrow R(X, \cdot) \cdot\}$.

Now we are ready to prove the following 
Theorem 3.1. If a connected trans-Sasakian 3-manifold of type $(\alpha, \beta)$ satisfies $\nabla \beta=\xi(\beta) \xi$, then one of the following cases occurs:

(1) $\alpha \neq 0, \beta \in \mathbb{R}^{*}$, and $\xi(\alpha) \neq 0$.

(2) $\alpha \neq 0, \beta=0$, and $\xi(\alpha)=0$.

(3) $\alpha=0, \beta \neq 0, \xi(\beta) \neq 0$, and $\nabla \xi(\beta)=\xi(\xi(\beta)) \xi$.

(4) $\alpha=0, \beta \in \mathbb{R}$.

Proof. If on a connected trans-Sasakian 3-manifold of type $(\alpha, \beta)$ there holds $\nabla \beta=$ $\xi(\beta) \xi$, taking the covariant derivative of this equation we have

$$
\nabla_{X} \nabla \beta=X(\xi(\beta)) \xi+\xi(\beta)(-\alpha \phi X+\beta X-\beta \eta(X) \xi)
$$

for any vector field $X$, where we have used (2.3). Note that the Hessian $H_{\beta}$ is a symmetric bilinear form defined by

$$
H_{\beta}(X, Y)=g\left(\nabla_{X} \nabla \beta, Y\right)
$$

for any vector fields $X, Y$. Thus, the inner product of $(3.1)$ with $Y$ gives

$$
H_{\beta}(X, Y)=X(\xi(\beta)) \eta(Y)-\alpha \xi(\beta) g(X, \phi Y)+\beta \xi(\beta) g(X, Y)-\beta \xi(\beta) \eta(X) \eta(Y) .
$$

Interchanging $X$ and $Y$ in the above equation gives

$$
H_{\beta}(Y, X)=Y(\xi(\beta)) \eta(X)-\alpha \xi(\beta) g(Y, \phi X)+\beta \xi(\beta) g(X, Y)-\beta \xi(\beta) \eta(X) \eta(Y) .
$$

In view of the symmetry of $H_{\beta}$, subtracting the above equation from the previous one gives

$$
X(\xi(\beta)) \eta(Y)-Y(\xi(\beta)) \eta(X)-2 \alpha \xi(\beta) g(X, \phi Y)=0
$$

for any vector fields $X, Y$. Putting $Y=\xi$ in 3.2 gives $X(\xi(\beta))-\xi(\xi(\beta)) \eta(X)=0$ for any vector field $X$. This is equivalent to $\nabla \xi(\beta)=\xi(\xi(\beta)) \xi$.

Let $X$ in $(3.2)$ be an arbitrary unit vector field orthogonal to the Reeb vector field $\xi$. Putting $Y=\phi X$ in 3.2 we obtain $\alpha \xi(\beta)=0$ and this implies that one of the following three cases occurs: $\alpha=0$ and $\xi(\beta) \neq 0$, or $\alpha \neq 0$ and $\xi(\beta)=0$, or $\alpha=\xi(\beta)=0$. Notice that when $\xi(\beta)=0$, by the assumption of the theorem we see that $\beta$ is a constant. The proof follows from Lemma 3.1 .

From equation $(2.3)$ we obtain $\operatorname{div} \xi=2 \beta$. Therefore, on any compact transSasakian 3-manifold, $\beta$ cannot be a non-zero constant. The following corollary follows directly from Theorem 3.1

Corollary 3.1. On a non-cosymplectic compact trans-Sasakian 3-manifold of type $(\alpha, \beta)$ satisfying $\nabla \beta=\xi(\beta) \xi$, we have either $\alpha \neq 0, \beta=0$ and $\xi(\alpha)=0$, or $\alpha=0$, $\beta \neq 0, \xi(\beta) \neq 0$ and $\nabla \xi(\beta)=\xi(\xi(\beta)) \xi$.

Corollary 3.1 proves the first conclusion of Theorem 1.2 Next, we need only to show a proof of the last conclusion of Theorem 1.2 namely we show that there exist many trans-Sasakian 3-manifolds which are of $C_{5}$-class with $\beta$ a non-constant function or of $C_{6}$-class with $\alpha$ a non-constant function. All the constructions in these examples depend on the following lemma. 
Lemma 3.3 ([18]). Let $(M, \phi, \xi, \eta, g)$ be a trans-Sasakian 3-manifold of type $(\alpha, \beta)$ and $f$ be a positive function on $M$. Then, $\left(M, \phi, \xi, \eta, g^{\prime}\right)$ is also a trans-Sasakian 3 -manifold of type $\left(\frac{\alpha}{f}, \beta+\frac{1}{2 f} \xi(f)\right)$, where the Riemannian metric $g^{\prime}$ is defined by $g^{\prime}=f g+(1-f) \eta \otimes \eta$.

Example 3.1. Let $(x, y, z)$ be the canonical Cartesian coordinates in $\mathbb{R}^{3}$. On $\mathbb{R}^{3}$ there exists a standard Sasakian structure (see Blair [2, p. 60]) defined as

$$
\begin{gathered}
\xi=2 \frac{\partial}{\partial z}, \quad \eta=\frac{1}{2}(d z-y d x) \\
\phi=\left(\begin{array}{ccc}
0 & 1 & 0 \\
-1 & 0 & 0 \\
0 & y & 0
\end{array}\right) \quad \text { and } g=\frac{1}{4}\left(\begin{array}{ccc}
1+y^{2} & 0 & -y \\
0 & 1 & 0 \\
-y & 0 & 1
\end{array}\right) .
\end{gathered}
$$

The orthonormal $\phi$-basis is given by $\left\{\xi, e_{1}:=2 \frac{\partial}{\partial y}, e_{2}:=\phi e_{1}=2\left(\frac{\partial}{\partial x}+y \frac{\partial}{\partial z}\right)\right\}$. Let $f$ be a positive function on $\mathbb{R}^{3}$. From Lemma 3.3 . $\left(\mathbb{R}^{3}, \phi, \xi, \eta, g^{\prime}\right)$ is a trans-Sasakian 3-manifold of type $\left(\frac{1}{f}, \frac{1}{2 f} \xi(f)\right)$, where $g^{\prime}=f g+(1-f) \eta \otimes \eta$.

Obviously, let $f=f(x, y)$ be a non-constant positive function on $\mathbb{R}^{3}$; then the non-compact trans-Sasakian manifold $\left(\mathbb{R}^{3}, \phi, \xi, \eta, g^{\prime}\right)$ of type $\left(\frac{1}{f}, 0\right)$ is of $C_{6}$-class but $\frac{1}{f}$ is not a constant and satisfies $\xi\left(\frac{1}{f}\right)=0$.

Example 3.2. Let $S^{3}$ be the standard unit sphere which is defined by

$$
S^{3}=\left\{(x, y, z, w) \in \mathbb{R}^{4}: x^{2}+y^{2}+z^{2}+w^{2}=1\right\} .
$$

On $S^{3}$ we consider the following three vector fields:

$$
\begin{aligned}
& e_{1}=-z \frac{\partial}{\partial x}+w \frac{\partial}{\partial y}+x \frac{\partial}{\partial z}-y \frac{\partial}{\partial w}, \\
& e_{2}=-w \frac{\partial}{\partial x}-z \frac{\partial}{\partial y}+y \frac{\partial}{\partial z}+x \frac{\partial}{\partial w}, \\
& e_{3}=-y \frac{\partial}{\partial x}+x \frac{\partial}{\partial y}-w \frac{\partial}{\partial z}+z \frac{\partial}{\partial w} .
\end{aligned}
$$

On $S^{3}$ there exists a standard Sasakian structure $\left(S^{3}, \phi, \xi, \eta, g\right)$ (see 2 and also [17, p. 158, Theorem 3.2]) as follows:

$$
\begin{gathered}
\xi=e_{1}, \quad \eta=g\left(e_{1}, \cdot\right), \\
\phi=\left(\begin{array}{ccc}
0 & 0 & 0 \\
0 & 0 & -1 \\
0 & 1 & 0
\end{array}\right) \quad \text { and } g=\left(\begin{array}{ccc}
1 & 0 & 0 \\
0 & 1 & 0 \\
0 & 0 & 1
\end{array}\right)
\end{gathered}
$$

with respect to the $\phi$-basis $\left\{e_{1}, e_{2}, e_{3}\right\}$. Let $f$ be a positive function on $S^{3}$. From Lemma 3.3 $\left(S^{3}, \phi, \xi, \eta, g^{\prime}\right)$ is a trans-Sasakian 3-manifold of type $\left(\frac{1}{f}, \frac{1}{2 f} \xi(f)\right)$, where $g^{\prime}=f g+(1-f) \eta \otimes \eta$.

Let $f$ be a non-constant positive function on $S^{3}$ satisfying the partial differential equation $-y \frac{\partial f}{\partial x}+x \frac{\partial f}{\partial y}-w \frac{\partial f}{\partial z}+z \frac{\partial f}{\partial w}=0$. For example, let be $f=\ln \left(x^{2}+y^{2}\right)$ or $f=\ln \left(z^{2}+w^{2}\right)$. Then, the compact trans-Sasakian 3-manifold $\left(S^{3}, \phi, \xi, \eta, g^{\prime}\right)$ of type $\left(\frac{1}{f}, 0\right)$ is of $C_{6}$-class but $\frac{1}{f}$ is not a constant and satisfies $\xi\left(\frac{1}{f}\right)=0$. 
From Examples 3.1 and 3.2 we have

Proposition 3.1. A trans-Sasakian 3-manifold $M$ of type $(\alpha, 0)$ is not necessarily $\alpha$-Sasakian even when the manifold is compact.

Example 3.3. Let $\mathbb{H}^{3}(-1)$ be the usual hyperbolic 3 -space which is defined by $\mathbb{H}^{3}(-1)=\left\{(x, y, z) \in \mathbb{R}^{3}: x>0\right\}$ with Riemannian metric $g=\frac{1}{x^{2}}(d x \otimes d x+d y \otimes$ $d y+d z \otimes d z)$. On $\mathbb{H}^{3}(-1)$ there exists a Kenmotsu structure given as (see [4]):

$$
\begin{gathered}
\xi=-x \frac{\partial}{\partial x}, \quad \eta=g(\xi, \cdot), \\
\phi \xi=0, \quad \phi \frac{\partial}{\partial y}=\frac{\partial}{\partial z}, \quad \phi \frac{\partial}{\partial z}=-\frac{\partial}{\partial y} .
\end{gathered}
$$

Let $f=f(x) \neq x^{c}, c$ a constant, be an arbitrary non-constant positive function defined on $\mathbb{H}^{3}$. Therefore, from Lemma 3.3 , the non-compact trans-Sasakian 3 -manifold $\left(\mathbb{H}^{3}, \phi, \xi, \eta, g^{\prime}\right)$ of type $\left(0,1-\frac{x}{2 f} \frac{\partial f}{\partial x}\right)$ is of $C_{5}$-class satisfying $\nabla \beta=\xi(\beta) \xi$ but neither $\beta$-Kenmotsu nor cosymplectic.

Example 3.4. Let $S$ be the usual unit circle $S=\left\{e^{i t}\right\}$ and $T^{2}$ be the torus with the standard Kähler structure $\left(J, g_{T^{2}}\right)$. Then, from [19], the product $\left(S \times T^{2}, g\right)$ with the Riemannian metric $g=d t \otimes d t+g_{T^{2}}$ is a cosymplectic 3-manifold with $\xi=\frac{\partial}{\partial t}$ and $\eta=d t$. Let $f=f(t) \neq e^{c t}, c$ a constant, be an arbitrary non-constant positive function defined on $S \times T^{2}$. Therefore, from Lemma 3.3 the compact transSasakian 3-manifold $\left(S \times T^{2}, \phi, \xi, \eta, g^{\prime}\right)$ of type $\left(0, \frac{1}{2 f} \frac{\partial f}{\partial t}\right)$ is of $C_{5}$-class satisfying $\nabla \beta=\xi(\beta) \xi$ but neither $\beta$-Kenmotsu nor cosymplectic.

From Examples 3.1 and 3.4 we have

Proposition 3.2. A trans-Sasakian 3-manifold $M$ of type $(0, \beta)$ satisfying $\nabla \beta=$ $\xi(\beta) \xi$ is not necessarily $\beta$-Kenmotsu or cosymplectic even when $M$ is compact.

Remark 3.1. The proof of Theorem 1.2 follows from Corollary 3.1 and Propositions 3.1 and 3.2

\section{ACKNOWLEDGEMENT}

The authors would like to thank the reviewer for his or her careful reading and useful suggestions.

\section{REFERENCES}

[1] N. Aktan, M. Yildirim and C. Murathan, Almost $f$-cosymplectic manifolds, Mediterr. J. Math. 11 (2014), no. 2, 775-787. MR 3198639

[2] D. E. Blair, Riemannian geometry of contact and symplectic manifolds, Second edition, Progress in Mathematics, Volume 203, Birkhäuser, 2010. MR 2682326

[3] D. E. Blair and J. A. Oubiña, Conformal and related changes of metric on the product of two almost contact metric manifolds, Publ. Mat. 34 (1990), no. 1, 199-207. MR 1059874 
[4] D. Chinea and C. Gonzalez, A classification of almost contact metric manifolds, Ann. Mat. Pura Appl. 156 (1990), 15-36. MR 1080209

[5] U. C. De and K. De, On a class of three-dimensional trans-Sasakian manifolds, Commun. Korean Math. Soc. 27 (2012), no. 4, 795-808. MR 3025885.

[6] U. C. De and A. Sarkar, On three-dimensional trans-Sasakian manifolds, Extracta Math. 23 (2008), no. 3, 265-277. MR 2524542

[7] U. C. De and M. M. Tripathi, Ricci tensor in 3-dimensional trans-Sasakian manifolds, Kyungpook Math. J. 43 (2003), no. 2, 247-255. MR 1982228

[8] S. Deshmukh, Trans-Sasakian manifolds homothetic to Sasakian manifolds, Mediterr. J. Math. 13 (2016), no. 5, 2951-2958. MR 3554289

[9] S. Deshmukh, Geometry of 3-dimensional trans-Sasakaian [i.e. trans-Sasakian] manifolds, An. Ştiinţ. Univ. Al. I. Cuza Iaşi Mat. (N.S.) 62 (2016), no. 1, 183-192. MR 3686608.

[10] S. Desmukh, U. C. De and F. Al-Solamy, Trans-Sasakian manifolds homothetic to Sasakian manifolds, Publ. Math. Debrecen 88 (2016), no. 3-4, 439-448. MR 3491752

[11] S. Deshmukh and F. Al-Solamy, A note on compact trans-Sasakian manifolds, Mediterr. J. Math. 13 (2016), no. 4, 2099-2104. MR 3530920

[12] S. Deshmukh and M. M. Tripathi, A note on trans-Sasakian manifolds, Math. Slovaca 63 (2013), no. 6, 1361-1370. MR 3146625

[13] A. Gray and L. M. Hervella, The sixteen classes of almost Hermitian manifolds and their linear invariants, Ann. Mat. Pura Appl. 123 (1980), 35-58. MR 0581924

[14] D. Janssens and L. Vanhecke, Almost contact structures and curvature tensors, Kodai Math. J. 4 (1981), no. 1, 1-27. MR 0615665

[15] K. Kenmotsu, A class of almost contact Riemannian manifolds, Tôhoku Math. J. 24 (1972), no. 1, 93-103. MR 0319102

[16] V. Mangione, Harmonic maps and stability on $f$-Kenmotsu manifolds, Int. J. Math. Math. Sci. 2008, Art. ID 798317, 7 pp. MR 2377360

[17] M. Markellos and C. Tsichlias, Contact metric structures on $S^{3}$, Kodai Math. J. 36 (2013), no. 1, 154-166. MR 3043406

[18] J. C. Marrero, The local structure of trans-Sasakian manifolds, Ann. Mat. Pura Appl. 162 (1992), 77-86. MR 1199647.

[19] Z. Olszak, On almost cosymplectic manifolds, Kodai Math. J. 4 (1981), no. 2, 239-250. MR 0630244

[20] Z. Olszak, Normal almost contact metric manifolds of dimension three, Ann. Polon. Math. 47 (1986), no. 1, 41-50. MR 0859423

[21] Z. Olszak, Locally conformal almost cosymplectic manifolds, Colloq. Math. 57 (1989), no. 1, 73-87. MR 1028604

[22] Z. Olszak and R. Roşca, Normal locally conformal almost cosymplectic manifolds, Publ. Math. Debrecen 39 (1991), no. 3-4, 315-323. MR 1154263

[23] J. A. Oubiña, New classes of almost contact metric structures, Publ. Math. Debrecen 32 (1985), no. 3-4, 187-193. MR 0834769

[24] Y. Wang, Minimal and harmonic Reeb vector fields on trans-Sasakian 3-manifolds, J. Korean Math. Soc. 55 (2018), no. 6, 1321-1336. MR 3883483

[25] A. Yildiz, U. C. De and M. Turan, On 3-dimensional f-Kenmotsu manifolds and Ricci solitons, Ukrainian Math. J. 65 (2013), no. 5, 684-693. MR 3150789 
Yaning Wang ${ }^{凶}$

School of Mathematics and Information Sciences, Henan Normal University, Xinxiang 453007, Henan, P. R. China

wyn051@163. com

Wenjie Wang

School of Mathematical Sciences, Dalian University of Technology, Dalian 116024, Liaoning, P. R. China

wangwj072@163.com

Received: December 15, 2017

Accepted: December 12, 2018 